\title{
Voto electrónico: confiabilidad y utilización de tecnología
}

\author{
Electronic voting: reliability and implementation of technology
}

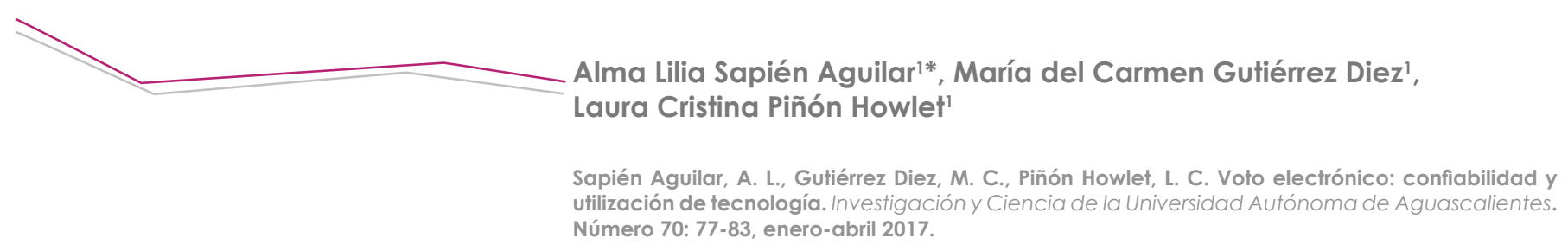

RESUMEN The population were Mexican voters, using opinion surveys applied by the Center for Social Studies and Public Opinion of the Chamber of Deputies. Being

El voto electrónico ha tomado importancia en México, ya que se han realizado estudios para su probable incorporación en las votaciones. En este trabajo se realizó un análisis de la confiabilidad y la utilización de la tecnología en este proceso, por su implicación social y económica. La población fueron los votantes mexicanos, mediante la encuesta de sondeos de opinión aplicada por el Centro de Estudios Sociales y de Opinión Pública (CESOP) en 2014. Se utilizó una muestra de 1,400 votantes con edad promedio de 38 años; la proporción de hombres y mujeres fue la misma. Entre los resultados más importantes destacan que la mayoría tiene credencial de elector vigente y considera que con el voto electrónico es más fácil manipular los resultados, pero si este se llegara a utilizar, consideran más confiable la votación presencial a través de urnas electrónicas instaladas en lugares adecuados.

\section{ABSTRACT}

Electronic voting has become important in Mexico, since there have been studies for inclusion in the voting in Mexico. This paper presents an analysis of reliability and the use of technology in this process, its social and economic implication was performed.

Palabras clave: voto electrónico, fiabilidad, costo y tecnología.

Keywords: electronic voting, reliability, cost and technology.

\section{Recibido: 1 de octubre de 2015, aceptado: 18 de julio de 2016}

1 Facultad de Contaduría y Administración, Universidad Autónoma de Chihuahua, México.

* Autor para correspondencia: Isapien@uach.mx the sample of 1,400 voters with an average age of 38 years, and the proportion of men and women was the same. Among the most important results it stands out that the majority of respondents have a valid electoral ID card, and believes that electronic voting is easier to manipulate the results, but if this was used, voting would be considered more reliable in person through electronic voting machines installed in suitable locations.

\section{INTRODUCCIÓN}

La aplicación de nuevas tecnologías en materia electoral ha aumentado a nivel mundial en los últimos años. El voto electrónico es un término que se ha empleado en forma general para identificar este proceso. De manera general existen dos tipos de voto electrónico. El primero se encuentra físicamente supervisado por representantes del órgano electoral y el segundo es el remoto, en el cual el votante no necesita desplazarse a una casilla electoral y se realiza mediante la tecnología de telefonía celular o por internet (Zissis y Lekkas, 2011).

El tema de voto electrónico remoto es mucho más complejo y actualmente pocos países se encuentran en posibilidades de implementarlo de forma masiva. Algunos otros muestran suspicacia a permitir que los ciudadanos emitan votos mediante internet y telefonía celular, pues se duda de la confiabilidad de los servidores que aplican y analizan los resultados $y$, por consiguiente, de la voluntad ciudadana. Los intentos de utilizar las tecnologías de la información y de las comunicaciones (TIC) en los diversos aspectos del voto electrónico (VE) 
pueden parecer recientes, pero no es así. De hecho, una de las primeras aplicaciones de las tecnologías electromecánicas sucedió a finales del siglo XIX, fue su uso para el ejercicio del VE y del recuento de votos posterior (Creelan y Norden, 2005).

La idea de modernizar los procesos electorales utilizando tecnologías basadas en la electrónica proviene de pensadores como Fromm (1955), Fuller (1963), Arterton (1987) y Rheingold (1993). En la actualidad existen pocos países que no han intentado desarrollar pruebas de voto electrónico con diversos tipos de soluciones y tecnologías. El objetivo de este trabajo fue analizar la confiabilidad y la utilización de la tecnología en el voto electrónico a partir de la encuesta de sondeos de opinión aplicada por el Centro de Estudios Sociales y de Opinión Pública (CESOP) en 2014.

\section{MATERIALES Y MÉTODOS}

Para el análisis de la base de datos se utilizó el programa estadístico SPSS (Statistical Package for the Social Sciences). Se realizaron análisis estadísticos descriptivos basados en la asociación de variables, tabulación cruzada, análisis de correlaciones, de frecuencias, correspondencias y chi-cuadrada con el objetivo de reportar los resultados de la investigación en forma simplificada y con alto grado de confiabilidad y validez estadística. Para el estudio de la información se utilizó el instrumento realizado por el Centro de Estudios Sociales y de Opinión Pública (CESOP) sobre el voto electrónico aplicado en 2014, se examinó lo relacionado con la utilización de tecnología, el conocimiento del voto electrónico y la confiabilidad. La población estuvo conformada por 76 millones de votantes, que eran los registrados en el padrón de votantes mexicanos del Instituto Nacional Electoral (INE). Se aplicaron 1,400 encuestas, para esto se utilizó muestreo aleatorio estratificado, proporcional a la población de cada estado y municipio.

RESULTADOS

Fueron analizadas diversas preguntas en relación con la confiabilidad que los votantes tienen con respecto al uso del voto electrónico, los costos de este sistema tecnológico: si se considera que sería un sistema más barato, igual o más caro que el sistema actual de votación y la utilización de tecnología. No hay relación entre la edad y la consideración de que el voto electrónico es o no confiable. La correlación es significativa pero hay poca fuerza en la relación de estas variables. De los encuestados, $62.4 \%$ prefieren la votación presencial a través de urnas electrónicas instaladas en lugares adecuados. Estos análisis estadísticos muestran diferentes niveles de asociaciones entre aspectos de la percepción de confianza y participación ante el uso de un voto electrónico, sus motivos para votar o no votar, así como la perspectiva ante la situación del país, índices de estado de ánimo social y público, interés en participar el procesos electorales, mismos que pueden ser observados a través de los diferentes grupos de edad establecidos para tal fin.

Un resumen de todos los aspectos analizados se muestra en la Tabla 1.

Si se empieza por analizar cuáles son los grupos que participan o no, de acuerdo con las elecciones presidenciales de 2012, se encuentra que los grupos más numerosos oscilan entre las edades desde 26 hasta 49 años, en total más de la mitad de los votantes; mientras el grupo que presenta mayor abstencionismo es el de edades comprendidas entre los 18 y los 25 años. Esto indica que entre los más jóvenes existe una falta de interés en participar.

Al analizar sus motivos para sí votar, se encuentran mayormente razones de confianza, utilidad e interés. A nivel país se establecieron razones técnicas y de confianza. Estos resultados contrastan con sus respectivas visiones de la situación del país. Para los grupos entre 26 y 49 años, son mayormente pesimistas y con miedo. De igual forma se perciben el Índice de Estado de Ánimo Social (IEAS) e Índice de Estado de Ánimo Público (IEAP), los cuales mostraron valores interpretados como negativos; en ambos casos de acuerdo a los resultados del estudio del CESOP (2014). En el caso de los más jóvenes, su percepción es optimista; sin embargo, son los que menos participan en las elecciones.

Por otra parte, para los grupos mencionados con mayor participación, su interés en los procesos electorales es prácticamente ninguno. Al evaluar la percepción de confiabilidad con respecto al uso del voto electrónico, lo ven como igual o más confiable que el sistema actual. De igual forma perciben de forma positiva el uso de esta tecnología del voto electrónico, para aumentar la participación en los comicios. Es interesante observar que el grupo que presenta mayor porcentaje de abstencionismo, los jóvenes de 18-25 años, percibe de forma contrastante 
Tabla 1. Grupos de edad y sus diferentes percepciones

\begin{tabular}{|c|c|c|c|c|c|c|c|c|c|}
\hline $\begin{array}{l}\text { Rango } \\
\text { edad }\end{array}$ & $\begin{array}{c}\text { Sí votó } \\
2012\end{array}$ & $\begin{array}{l}\text { Razones } \\
\text { sí votó }\end{array}$ & $\begin{array}{l}\text { No } \\
\text { votó } \\
1012\end{array}$ & $\begin{array}{l}\text { Razones } \\
\text { no votó }\end{array}$ & $\begin{array}{l}\text { Situación de } \\
\text { país }\end{array}$ & $\begin{array}{l}\text { Interés en } \\
\text { procesos } \\
\text { electorales }\end{array}$ & $\begin{array}{c}\text { Confianza } \\
\text { voto } \\
\text { electrónico }\end{array}$ & $\begin{array}{l}\text { Participación } \\
\text { con voto } \\
\text { electrónico }\end{array}$ & Medios \\
\hline $18-25$ & 124 & $\mathrm{~T} / \mathrm{I}$ & 200 & $U / I$ & Optimista & & $\begin{array}{l}\text { Menos } \\
\text { confiable }\end{array}$ & $\begin{array}{c}\text { Más } \\
\text { participación }\end{array}$ & Internet/RS \\
\hline $26-33$ & 161 & $\mathrm{U} / \mathrm{C}$ & 80 & $\mathrm{U} / \mathrm{C}$ & Pesimista & & $\begin{array}{c}\text { Igual } \\
\text { confiable }\end{array}$ & $\begin{array}{c}\text { Más } \\
\text { participación }\end{array}$ & Internet/RS \\
\hline $34-41$ & 157 & $C / I$ & 80 & $\mathrm{C} / \mathrm{I}$ & Pesimista & Nada & $\begin{array}{c}\text { Igual } \\
\text { confiable }\end{array}$ & $\begin{array}{c}\text { Igual } \\
\text { participación }\end{array}$ & Internet/RS \\
\hline $42-49$ & 170 & $\mathrm{I} U$ & 90 & $\mathrm{l} / \mathrm{T}$ & Con miedo & Nada & Más confiable & & Internet/RS \\
\hline $50-57$ & 109 & $\mathrm{I} / \mathrm{T}$ & 36 & $\mathrm{C} / \mathrm{T}$ & Optimista & Mucho & $\begin{array}{c}\text { Menos } \\
\text { confiable }\end{array}$ & $\begin{array}{c}\text { Igual } \\
\text { participación }\end{array}$ & Internet/RS \\
\hline $58-65$ & 47 & $T / P$ & 16 & $C / T$ & Esperanza & & $\mathrm{Nc}$ & & Internet/RS \\
\hline $66-73$ & 29 & $T / P$ & 11 & $\mathrm{C} / \mathrm{T}$ & Triste & & & & Internet/RS \\
\hline $74-87$ & 16 & $U / C$ & 2 & $T / I$ & $\mathrm{Ns} / \mathrm{Nc}$ & & & & Internet/RS \\
\hline $\begin{array}{l}\text { Total } \\
\text { país }\end{array}$ & 813 & $\mathrm{~T} / \mathrm{C}$ & 515 & $\mathrm{P} / \mathrm{T}$ & & & & & \\
\hline
\end{tabular}

T-técnicas, l-interés, U-utilidad, C-confianza, P-personales, RS-redes sociales, Ns-no sabe, Nc-no contestó. Gutiérrez Diez y Salcido Ornelas (2015).

una mayor participación en las elecciones al usar el voto electrónico, aunque no lo perciben como confiable. Quizá esto pudiera explicarse por la curiosidad propia de experimentar con la nueva modalidad.

Acerca de los motivos de quienes respondieron no haber participado en las últimas elecciones presidenciales, y en cuanto a las correlaciones, mostraron valores contundentes, al detallar las respuestas para las razones que tuvieron la mayoría de los encuestados dijeron no saber o no contestaron, lo cual no permite establecer claramente los motivos de dicho abstencionismo. Sin embargo, al continuar analizando los porcentajes se encuentra que el motivo personal que describe que porque se dio cuenta que el voto se intercambia por favores, dinero o regalos es el de mayor frecuencia. Para los grupos restantes, con edades entre 58 y 87 años, aunque participan, no muestran ninguna asociación o relación en particular.

Otra observación es el hecho de que los grupos de edad más jóvenes, entre los 18 y 57 años, tienen la percepción de una mayor participación, así como de confianza en el uso del voto electrónico, independientemente de su poco interés en participar en elecciones. Su percepción varía en la confianza hacia el uso de esta tecnología. El porcentaje que considera más barato usar el voto electrónico en relación con el sistema actual es $43.1 \%$, pero $32.7 \%$ lo considera más caro.

Se afirma que la participación ciudadana es un factor clave en los procesos de fortalecimiento democrático, en particular en América Latina su formación y evolución ha sido creciente en los últimos años. Algunos cambios legales han permitido la inclusión de los ciudadanos en las tomas de decisiones y con esto el ejercicio de soberanía más allá del voto. En el caso de México, según la Encuesta Mundial de Valores (World Values Survey Association, 2005), $76 \%$ de los mexicanos aseguran no pertenecer a ningún tipo de organización; solamente $13.8 \%$ afirmó ser miembro activo de alguna, principalmente relacionada con religión, deporte y recreación (Vergara Lope Tristán y Hevia de la Jara, 2012).

Esta pertenencia o asociación se puede explicar levemente según el Informe país sobre la calidad de la ciudadanía en México, publicado en 2015 por el INE (Instituto Nacional Electoral) y el COLMEX (Colegio de México), a través de la confianza. Si la persona confía tiene mayor probabilidad de relacionarse e incrementar su participación en diferentes tipos de procesos y en la toma de decisiones derivada de los mismos. 
Si se analiza la opinión expresada por los ciudadanos en la encuesta del CESOP (2014), donde se les preguntó la opinión con respecto a las instituciones y autoridades, como el presidente, partido en el poder, representantes de partidos, gobierno de la república, poder legislativo, tribunales y juzgados; se encuentra que la balanza se inclina hacia una mala y muy mala percepción, lo cual demerita la confianza hacia las mismas y su propio involucramiento con ellas.

Entonces, ¿̇uál es esa pertenencia a una comunidad?, ¿existe en México este sentido de pertenencia? De acuerdo al estudio ya mencionado de calidad de la ciudadanía de 2015, los resultados sugieren que la mayor parte de los municipios del país son de bajo desarrollo, por lo que no hay condiciones que favorezcan las asociaciones locales y, por tanto, existe una fragmentación de la sociedad, lo cual no permite la generación de una comunidad que participe y exija sus derechos.

Por otra parte, existen índices que permiten establecer cuál es el estado de ánimo social y público. Según establece el CESOP(2014), en los resultados de la encuesta nacional de opinión pública acerca de voto electrónico: "el Índice estado de Ánimo social (IEAS), similar al índice social mood, mide qué tan optimistas o pesimistas se sienten los ciudadanos respecto lo que resta del sexenio". Otro registro establecido para el mismo estudio es el índice de estado de ánimo público (IEAP) derivado de cuatro indicadores, utilizado en el American National Election Studies, para tratar de entender las relaciones entre varios procesos afectivos y el comportamiento político de los ciudadanos.

\section{DISCUSIÓN}

De acuerdo con los hallazgos planteados, tanto de este estudio como de otros, se puso de manifiesto el impacto trascendente de las tecnologías de la información en diferentes aspectos de la sociedad y en las organizaciones, efectos de innovación, sociales y económicos; el voto electrónico es motivo de investigación y se han realizado diversos estudios que analizan la conveniencia o inconveniencia para su incorporación en la democracia, en las votaciones para elegir a los gobernantes en México. Con la idea de ayudar en la decisión del ingreso de este sistema electoral, en el presente trabajo se realizó un análisis en relación con su confiabilidad y cómo consideran los votantes la utilización de la tecnología

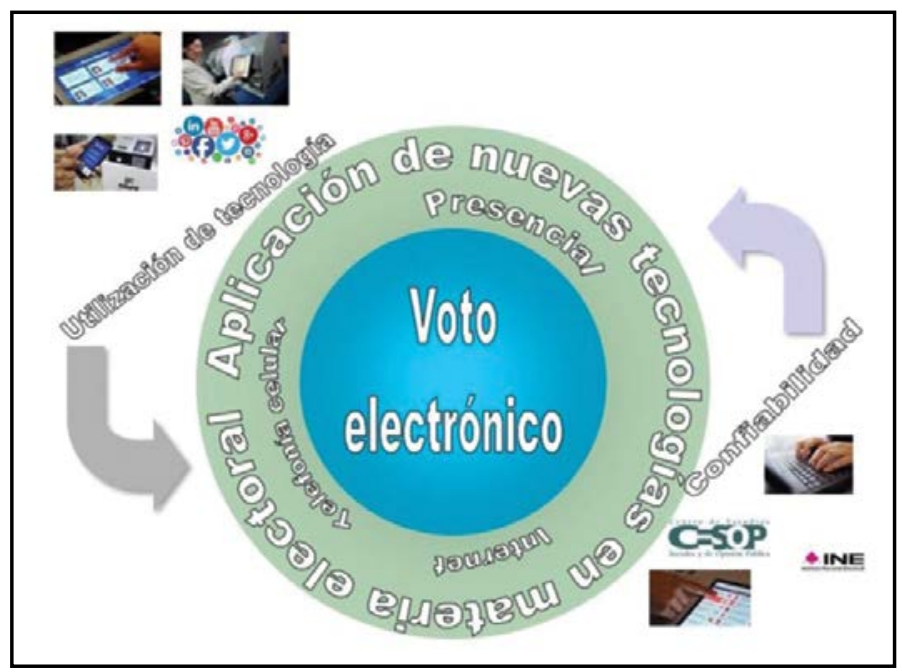

Figura 1. Voto electrónico: confiabilidad y utilización de tecnología. Imagen elaborada por Alma Lilia Sapién Aguilar.

en este proceso. Por tanto, la exigencia por producir transparencia, seguridad y disminución de los costos en los procesos electorales ha encontrado en la utilización de la tecnología un método que permite mejorar su realización. Sin embargo, el panorama de la legislación electoral europea parece un mosaico variado y colorido de requisitos, procedimientos y herramientas técnicas. Algunos países revocaron el voto electrónico cuando perdieron el apoyo de la base electoral, sin importar si era una novedad como en Irlanda (Commission on Electronic Voting, 2004a y b) o un hábito bien establecido como en Holanda (Gonggrijp et al., 2006; Oostveen, 2007, 2010). Otros han sido más cuidadosos y han organizado pruebas y experimentos con y sin valor legal, aunque siempre lo han hecho a una escala limitada; esto ha pasado en Suiza (Braun, 2004; Braun y Brändli, 2006), Gran Bretaña (Fairweather y Rogerson, 2003), España (Fernández Rodríguez et al., 2007), Portugal (Falcão e Cunha et al., 2008), e Italia (Caporusso, 2008). Actualmente algunos países, como Bélgica y Francia, utilizan máquinas electrónicas; mientras que en los países bálticos han explorado y adoptado canales más y más innovadores: voto por internet que se ha usado con éxito en Estonia (Madise y Martens, 2006) y ha sido considerado en Lituania (Udris, 2006), e incluso voto por mensaje de texto a través del teléfono celular, como fue aprobado recientemente en Estonia (World E-Democracy Forum, 2008).

Experiencias de voto vía internet de carácter legal muestran resultados contradictorios: encuestas realizadas después de las elecciones democráticas primarias en Arizona en el año 2000 llegaron a la 


\section{InVESTIGACIÓn Y CIERCIA DE LA UחIVERSIDAD AUTÓnOMA DE RGUASCALIEחTES}

misma conclusión, ya que encontraron un impacto significativo en edad y nivel de educación; mientras que el sexo no formó parte de la decisión de votar en línea (Solop, 2001; Kenski, 2005). Por otro lado, divergen de manera considerable al interpretar el efecto del ingreso, significativo a nivel de dos variables (Solop, 2001; Kenski, 2005) o al cruzar datos ecológicos en vez de individuales (Gibson, 2005); pero pierden poder al ser agrupados en un modelo multivariado (Solop, 2001). La ubicación (urbana/rural) no tiene un efecto estadísticamente significativo (Solop, 2001), ni la adherencia a un partido (Kenski, 2005). Algunos autores insisten en la existencia de una diferencia digital entre clases sociales y grupos de sexo y edad diferentes (Gibson, 2005). Datos individuales de participación electoral durante las elecciones primarias en Michigan del año 2004 les permitieron a los investigadores abordar las particulares preocupaciones de los defensores. La raza y clase no fueron significativas y un modelo de dos decisiones clarificó que su impacto es limitado a la opción de votar en ausencia: cuando se ha tomado esta decisión, no juegan un papel al seleccionar el método preferido (correo o internet) para votar (Prevost y Schaffner, 2008).

Existen otros estudios que refuerzan esta percepción de desconfianza y desencanto hacia el sistema político en general y en particular a los responsables de la procuración de justicia (CESOP, 2014; INE-COLMEX, 2015), aunque hay que destacar la variación de la confianza en organismos como el IFE/INE, que ha perdido credibilidad, ya que alcanzó su nivel máximo en el año 2000 durante la alternancia del PRI al PAN pero registró 9\% en 2011 (Martí I. Puig, 2012); mientras que en la encuesta de CESOP (2014), el nivel de credibilidad se reportó como $10 \%$.

Por otra parte, en estudios de opinión pública en democracias occidentales, se llega a la conclusión de que los ciudadanos cuentan con poca información acerca de los asuntos públicos, con opiniones contradictorias y poca estabilidad en sus juicios. Sin embargo, los mexicanos emiten continuamente opiniones sobre prácticamente cualquier cosa, incluyendo políticas públicas. Lo anterior se puede confirmar en los resultados obtenidos por el CESOP (2014), donde se expresa claramente que la principal fuente de información y noticias con respecto a lo que pasa en el país es a través de medios electrónicos: internet y redes sociales.

No se puede hablar de ciudadanía, ni sus percepciones acerca de cualquier tema, sin relacionarla con la participación. Para ello es fundamental distinguir entre la participación política y la que no lo es; aunque en muchas ocasiones esto causa confusión, es difícil distinguir una de la otra, e inclusive se desconoce que se puede participar políticamente sin participar electoralmente (en votaciones).

Es interesante observar que las asociaciones religiosas, a pesar de no participar a través de la emisión de un voto en las elecciones, sí participan o se mantienen informadas con una frecuencia diaria a través de los medios electrónicos, particularmente de las redes sociales. Estos medios ayudan a compensar la falta de información del ciudadano común, para que construya sus propias opiniones, de tal forma que pueda comprender la complejidad de los temas políticos (Buendía, 2010).

De acuerdo con los hallazgos planteados, tanto de este estudio como de otros, se coincide en que existe una carencia de cohesión y participación social, lo cual no permite la construcción de una ciudadanía activa, que ejerza y exija sus derechos. Existe una manifiesta desconfianza en instituciones y sus respectivos representantes. Lo peor es que tampoco se observa interés de los ciudadanos en participar en la toma de decisiones que conlleven a un cambio. Hay un desencanto total (Reyes, 2014).

Sin embargo, se puede esperar que la edad, sexo, ocupación y educación estén asociados con la inclinación a votar en internet o por medios electrónicos, según losresultados de esta investigación. Como explican Guerra y Zizzo (2003), la confianza en el otro es crucial al establecer relaciones, se argumenta que el flujo de confianza comienza al confiar en las instituciones que organizan las elecciones (Xenakis y Macintosh, 2005).

\section{CONCLUSIONES}

La mayoría de los encuestados consideró más confiable la votación presencial a través de urnas electrónicas instaladas en lugares adecuados. Al analizar los grupos de edad que participan 0 se abstienen en los procesos electorales, es alarmante observar el poco interés que muestran los segmentos de menor edad. Esta confianza tiene influencia de la percepción de la situación del país, reflejada en los índices establecidos como el estado de ánimo social y público. Este último es el que muestra mayor peso en la percepción de participación mediante el voto 
electrónico. Esta misma confianza establecida ante los organismos y mecanismos electorales se refleja en los motivos que llevan al ciudadano a participar en las elecciones y, por ende, a la aceptación eventual de los medios tecnológicos que se establezcan para recabar los votos.

Quienes tienen una percepción favorable de la situación del país muestran una opinión positiva ante el uso del voto electrónico, como una tecnología que permita recabar los sufragios y poder obtener los derivados del uso de esta tecnología. Por otra parte, si se retoma el análisis hecho a los medios de comunicación utilizados para conocer lo que sucede en el país, es posible observar que las barreras en el uso de la tecnología prácticamente no existen entre los diferentes grupos de edad, ya que todos coinciden en el uso de internet y de las redes sociales para informarse. A través de estos medios se debe mantener informada a la ciudadanía en los aspectos de confianza y participación activa en la vida política del país.

\section{LITERATURA CITADA}

- ARTERTON, C. Teledemocracy: can technology protect democracy? Newbury Park, California, US: SAGE publications, 1987.

- BRAUN, N. E-voting: Switzerland's projects and their legal framework. In A. PROSSER \& R. KRIMMER (Ed.), Electronic voting in Europe: Technology, law, politics and society. Workshop of the ESF TED Programme together with GI and OCG. July, $7^{\text {th-9th }}$ 2004 in SchloB Hofen/Bregenz, Lake of Constance, Austria (4352). Bonn, Germany: Gl, 2004.

- BRAUN, N. y BRÄNDLI, D. Swiss e-voting pilot projects: Evaluation, situation analysis and how to proceed. In R. KRIMMER (Ed.), Electronic voting 2006, Gl lecture notes in informatics (27-36). Bonn, Germany: Gl, 2006.

- CAPORUSSO, L. There is more to e- than meets the eye: Towards automated voting in Italy. In J. M. RENIU (Ed.), E-voting: The last electoral revolution (27-44). Barcelona, Spain: ICPS, 2008.

- FAIRWEATHER, B. y ROGERSON, S. Internet voting-Well at least it's modern. Representation, 39(3): 182-195, 2003.

- falCÃO E CUNHA, J. et al. Auditing e-voting pilot processes and systems at the elections for the European Parliament and for the Portuguese Parliament. In J. M. RENIU (Ed.), E-voting: The last electoral revolution (93-114). Barcelona, España: ICPS, 2008.

- FERNÁNDEZ RODRÍGUEZ, J. J. et al. Voto electrónico. Estudio comparado en una aproximación jurídico-política (desafíos y posibilidades). Santiago de Querétaro, México: Fundación Universitaria de Derecho, Administración y Política, 2007.

- FROMM, E. The sane society. New York, US: Rinehart, 1955.

- FULLER, B. R. No more secondhand God. US: Southern Illinois University Press, 1963.
- GUTIÉRREZ DIEZ, M. C. y SALCIDO ORNELAS, D. Factores que influyen sobre percepciones de confianza y participación en elecciones a través del voto electrónico. Chihuahua, México: Universidad Autónoma de Chihuahua, 2015.

- GIBSON, R. Internet voting and the European Parliament's elections: Problems and prospects. In A. TRECHSEL \& F. MENDEZ (Eds.), The European Union and e-voting: Addressing the European Parliament's internet voting challenge. London, UK: Routledge, 2005.

- KENSKI, K. To I-vote or not to I-vote? Opinions about internet voting from Arizona voters. Social Science Computer Review. 23(3): 293-303, 2005.

- MADISE, Ú. y MARTENS, T. E-voting in Estonia 2005. The first practice of country-wide binding internet voting in the world. In R. KRIMMER (Ed.), Electronic voting 2006, Gl lecture notes in informatics (15-26). Bonn, Germany: Gl, 2006.

- OOStVeen, A. M. Context matters. A social informatics perspective on the design and implications of large-scale e-government systems. Amsterdam, Netherlands: University of Amsterdam, 2007.

- OOSTVEEN, A. M. Outsourcing democracy: losing control of e-voting in the Netherlands. Policy \& Internet, 2(4): 201-220, 2010.

- PREVOST, A. K. y SCHAFFNER, B. F. Digital divide or just another absentee ballot? Evaluating internet voting in the 2004 Michigan democratic primary. American Politics Research, 36(4): 510-529, 2008

- RHEINGOLD, H. The virtual community. Homesteading on the electronic frontier. Reading, MA, US: Addison-Wesley, 1993.

- SOLOP, F. I. Digital democracy comes of age: Internet voting and the 2000 Arizona democratic primary election. PS: Political Science \& Politics, 34(2): 289-293, 2001. 
- UDRIS, J. The Lithuanian concept of voting via internet for elections and referenda. Presentation held at the Council of Europe on account of the Central Electoral Commission of the Republic of Lithuania, November 16, in Strasbourg, France. Bonn, Germany: Gl, 2006.

- XENAKIS, A. y MACINTOSH, A. Trust analysis of the UK e-voting pilots. Social Science Computer Review, 23(3): 312-325, 2005.

- ZISSIS, D. y LEKKAS, D. Securing e-Government and e-voting with an open cloud computing architecture. Goverment Information Quarterly, 28(2): 239-251, 2011.

\section{De páginas electrónicas}

- BUENDÍA, J. Los fundamentos de la opinión pública. En El Colegio de México (Ed.), Los grandes problemas de México: XIV. Instituciones y procesos políticos (303-330). 2010. Recuperado en febrero de 2015, de http://2010.colmex.mx/ tomos2.html

- CESOP (CENTRO DE ESTUDIOS SOCIALES Y DE OPINIÓN PÚBLICA DE LA CÁMARA DE DIPUTADOS). Encuestas. Diciembre de 2014. Recuperado en febrero de 2015, de http://www5.diputados. gob.mx/index.php/camara/Centros-de-Estudio/CESOP/ Opinion-Publica/Encuestas

- COMMISSION ON ELECTRONIC VOTING. First report of the Commission on Electronic Voting on secrecy, accuracy and testing of the chosen electronic voting system. 2004a. Recuperado de http://www.umic.pt/images/stories/ publicacoes 1/00Index.pdf

- COMMISSION ON ELECTRONIC VOTING. Interim report of the Commission on Electronic Voting on secrecy, accuracy and testing of the chosen electronic voting system. 2004b. Recuperado de http://www.umic.pt/images/stories/ publicacoes 1/V02.pdf

- CREELAN, J. y NORDEN, L. The requirements of New York University School of Law. Noviembre 2 de 2005. Recuperado de www.wheresthepaper.org/

- GONGGRIJP, R. et al. Nedap/Groenendaal ES3B voting computer. A security analysis. Spoedcursus stemcomputers. Wij vertrouwen stem computers niet. October 2006. Recuperado de http://wijvertrouwenstemcomputersniet.nl/other/es3b-en. pdf

- Guerra, G. A. y ZIzzO, D. J. Economics of trust in the information economy: Issues of identity, privacy and security. Research Report No. 1. Oxford, UK: Oxford Internet Institute, 2003. Recuperado de https://www.oii.ox.ac.uk/archive/ downloads/publications/RR1.pdf

- INE (INStituto NACIONAL ElECtORAL)-COlMEX (EL COLEGIO DE MÉXICO). Informe país sobre la calidad de la ciudadanía en México. 2015. Recuperado en febrero de 2015, de http:// www.ine.mx/archivos2/s/DECEYEC/EducacionCivica/ Resumen_Ejecutivo_23nov.pdf

- MARTí I. PUIG, S. Ciudadanía y cultura política en México a dos sexenios de la "alternancia". Foro Internacional, LII(4): 864-884, 2012. Recuperado de http://www.redalyc.org/ pdf/599/59929086005.pdf

- REYES, A. El reto de los valores, percepciones y prácticas ciudadanas: Informe País. El Semanario, 23 de agosto de 2014. Recuperado en febrero de 2015, de http://elsemanario. com/69606/el-reto-de-los-valores-percepciones-y-practicasciudadanas-informe-pais/

- VERGARA LOPE TRISTÁN, S. y HEVIA DE LA JARA, F. J. Para medir la participación. Construcción y validación del Cuestionario Conductas de Participación (CCP). Revista Mexicana de Ciencias Políticas y Sociales (UNAM), LVII(215): 35-67, 2012. Recuperado en 2014, de http://www.redalyc.org/articulo. oa? id $=42125325002$

- WORLD E-DEMOCRACY FORUM (2008). Estonia to vote by mobile phone in 2011. Recuperado de http://www. edemocracy-forum.com/2008/12/estonia-to-vote-bymobilephone-in-2011.html

- WVS (WORLD VALUES SURVEY ASSOCIATION). World Values Survey Wave 5 2005-2008 Official Aggregate v.20140429. Aggregate File Producer: Asep/JDS, Madrid, Spain, 2005. Recuperado de www.worldvaluessurvey.org 